\title{
Psicologia ambiental e saúde na relação pessoa-ambiente: uma revisão sistemática
}

Psicología ambiental y salud en la relación personal-ambiente: una revisión sistemática Environmental psychology and health in the people-environmental relationship: a systematic review

Bettieli Barboza da Silveira

ORCID: http://orcid.org/0000-0002-1935-3004 Universidade Federal de Santa Catarina, Santa Catarina/Brasil

Ariane Kuhnen

ORCID: http://orcid.org/0000-0001-9635-9306 Universidade Federal de Santa Catarina, Santa Catarina/Brasil

Declaração de Direito Autoral

A submissão de originais para este periódico implica na transferência, pelos autores, dos direitos de publicação impressa e digital. Os direitos autorais para os artigos publicados são do autor, com direitos do periódico sobre a primeira publicação. Os autores somente poderão utilizar os mesmos resultados em outras publicações indicando claramente este periódico como o meio da publicação original. Em virtude de sermos um periódico de acesso aberto, permite-se o uso gratuito dos artigos em aplicações educacionais e científicas desde que citada a fonte conforme a licença CC-BY da Creative Commons.

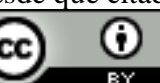

Creative Commons Atribuiç̃ão 4.0 Internacional.

\section{Resumo}

Ao almejar compreender a aplicação da psicologia ambiental na esfera da saúde se propõe contemplar as relações que as pessoas estabelecem com os lugares que habitam. Objetiva-se, portanto, revisar sistematicamente a literatura a fim de examinar os conceitos de ambiente restaurador, significado ambiental e identidade social urbana em relação com contextos de saúde. Essa tríade contempla a interação do sujeito com os espaços, enfatizando, sobretudo, a busca pelo bem-estar. Para tanto, foi realizada uma revisão sistemática da literatura nas bases de dados BVS-PSI, Scielo, Lilacs, Pepsic. Além disso, contemplou-se as bases de dados internacionais que dão acesso às principais revistas da Psicologia Ambiental, sendo elas: Science Direct e Sage Journals. Resultaram vinte sete estudos, analisados e subdivididos em três categorias estruturadas de modo a contemplar as diferenças temáticas, teóricas e metodológicas. Embora tenham delimitações distintas, os artigos examinados se comunicam entre si por meio da perspectiva comum de investir na busca de estratégias de melhoria da saúde e promoção de saúde considerando a relação com o ambiente. Desse modo, espera-se que os achados científicos ofereçam indicadores e subsídios no que condiz à avaliação e estruturação de ações e planejamentos ambientais em contextos de atenção à saúde.

Palavras-chaves: Psicologia ambiental; Saúde; Ambiente restaurador; Identidade social urbana; Significado ambiental.

\section{Resumen}

Al desear entender la aplicación de la psicología ambiental en la esfera de la salud se propone contemplar las relaciones que la gente establece con los lugares que habitan. El objetivo es, por lo tanto, revisar sistemáticamente la literatura para examinar los conceptos de entorno restaurativo, significación medioambiental e identidad social urbana en relación con los contextos de salud. Esta tríada contempla la interacción del sujeto con los espacios, enfatizando, sobre todo, la búsqueda del bienestar. Para ello, se realizó una revisión sistemática de la bibliografía en las bases de datos BVS- 
PSI, SciELO, LILACS, PePSIC. Además, se contemplaron las bases de datos internacionales que dan acceso a las principales revistas de psicología ambiental: Science Direct y Sage Journal. Resultaron en veintisiete estudios, analizados y subdivididos en tres categorías estructuradas para contemplar las diferencias temáticas, teóricas y metodológicas. Aunque tienen distintos límites, los artículos examinados se comunican entre sí a través de la perspectiva común de invertir en la búsqueda de estrategias de mejoramiento de la salud y promoción de la salud considerando la relación con el medio ambiente. De esta manera, se espera que los hallazgos científicos proporcionen indicadores y subsidios en la evaluación y estructuración de las acciones ambientales y la planificaciónen el contexto de la atención de la salud.

Palabras claves: Psicología ambiental; Salud; Ambiente restaurador; Identidad social urbana; Significado ambiental.

\begin{abstract}
In desiring to understand the application of environmental psychology in the sphere of health proposes to contemplate the relationships that people establish with the places they inhabit. It is therefore objective to systematically revise literature in order to examine the concepts of restorative environment, environmental significance and urban social identity in relation to health Contexts. This triad contemplates the interaction of the subject with the spaces, emphasizing, above all, the search for Well-being. In order to do so, a systematic review of the literature was carried out in the BVS-PSI, Scielo, Lilacs, Pepsic data bases. In addition, the international databases that give access to the main journals of environmental psychology were contemplated: Science Direct and Sage journals. They resulted in twenty seven studies, analyzed and subdivided into three structured categories in order to contemplate the thematic, theoretical and methodological differences. Although they have distinct boundaries, the articles examined communicate with each other through the common perspective of investing in the search for health improvement strategies and health promotion considering the relationship with the Environment. In this way, scientific findings are expected to provide indicators and subsidies in the assessment and structuring of environmental actions and planning in the context of health care.
\end{abstract}

Keywords: Environmental psychology; Health; Restorative environment; Urban social identity; Environmental meaning.

\section{Introdução}

Trata-se de um ambiente restaurador aquele com potencial de restaurar recursos e capacidades emocionais e funcionais comprometidas pelo estresse ou demandas cotidianas (Ulrich, 1984). Hartig e Staats (2005) reforçam que alguns ambientes podem melhor facilitar a restauração do que outros. Nesta direção, ao discorrer acerca da implicação de certos lugares à saúde humana, Corraliza (1998, p. 59) defende que "um dos processos mais relevantes da interação indivíduo-ambiente é constituído por aquele através do qual o espaço físico se converte em um espaço significativo para um indivíduo”. A interface proposta por meio dos conceitos de ambiente restaurador e de significado ambiental se amplia a fim de contemplar a implicação do sujeito perante o espaço que habita, ressaltando a importância dada ao processo de constituição de identidade social urbana.

Ao compreender a relevância de se explorar a tríade de conceitos propostos (ambiente restaurador - significado ambiental - identidade social urbana), parte-se da intenção de identificar e mapear elementos específicos que exercem influência positiva ou negativa sobre a saúde, especificamente sobre o estresse e o bem-estar, para futuramente intervir junto a eles. Para tanto, o contexto espacial delimitado para investigação nessa 
pesquisa visa percorrer Serviços de Residenciais Terapêuticos (SRT's), isto é, instituições em formato de casas que acolhem pessoas com deficiências ou doenças mentais, e atuam estrategicamente como dispositivos que acolhem pacientes egressos ou não de hospitais psiquiátricos (Ministério da Saúde, 2004). Para Silva e Azevedo (2011), nesse enfoque de tratamento de saúde mental se busca resgatar a autonomia dos internos, encorajando-os a assumir postura ativa no processo de conquista de espaço em sociedade. Desse modo, torna-se uma tarefa fundamental aclarar os conceitos explorados nessa revisão, assim como as características dos espaços envolvidos.

Compreendidas como as duas principais frentes de entendimentos sobre os ambientes restauradores, as teorias de Roger Ulrich (1984) - Psychophysiological Stress Recovery, e a de Rachel e Stephen Kaplan (1989) - Attention Restoration Theory, evidenciam e concordam sobre a influência dos ambientes para a saúde humana, por meio de diferentes condições antecedentes e por intermédio de diferentes processos. A restauração do estresse é um processo que ocorre quando a recuperação ou renovação dos recursos psicológicos, fisiológicos e sociais foi comprometida, tendo em vista as transformações e exigências da vida cotidiana (Hartig, 2011). Assim sendo, o processo de restauração pode ser promovido ou permitido pela presença de ambientes restauradores, isto é, um lugar que suscite interesse, aprazibilidade e calma (Ulrich, 1984). Desse modo, restaurtar o estresse se trata do retorno à normalidade dos aspectos piscofisiológicoss alterados pelas demandas diárias. Importante destacar que tais reações são fortemente mediadas pelas características visuais do ambiente, que, por sua vez, evocam respostas emocionais positivas e limitam distrações negativas, reestabelecendo equilíbrio ao sistema psicofisiológico alterado pelo estresse (Hartig, 2011; Felippe, Kuhnen, Silveira, \& Lelli, 2017).

Comumente associado em investigações sobre ambientes restauradores, o significado ambiental é compreendido pelas reações que as pessoas exprimem frente aos ambientes, de acordo com os significados construídos anteriormente em conexões com o meio (Rapoport, 1990). Atua como um modelo de comunicação não verbal, em que a pessoa identifica, interpreta e atribui sentido aos aspectos físicos e sociais que fazem parte da sua experiência de vida e, uma vez que tais informações são codificadas e esses aspectos se repetem, os mesmos sentidos são evocados, podendo conduzir seus modos de ação sobre o meio (Felippe et al., 2017; Rapoport, 1990). Nesse viés, o conceito de identidade social urbana se aproxima, haja vista sua estrutura direcionada a compreender a necessidade das pessoas identificarem territórios como próprios, assim como a importancia atribuída aos vínculos emocionais estabelecidos com o entorno (Mourão \& Cavalcante, 2006; Pol, 1996). Para Valera e Pol (1994), trata-se da transição na percepção de um espaço indiferenciado transformado em lugar na medida em que a pessoa o experimenta e o vivencia através do tempo e da intensidade, passando a ser percebido com valor afetivo.

Ao discorrer sobre espaços e lugares, torna-se de grande valia identificar a trajetória e as características dos ambientes investigados, sendo assim, faz-se um adendo ao percurso do dispositivo de atenção à saúde que hoje chamamos de SRT. O modelo hospitalocêntrico e os movimentos populares impulsionaram fortemente a Reforma Psiquiátrica no Brasil ainda na década de 1970, até que em 2001, a Lei 10.216 foi sancionada, redirecionando a assistência em saúde mental no país (Yasui, 2010). Desde então, embora esteja ausente na Lei um melhor detalhamento sobre os mecanismos para a progressiva extinção dos manicômios, um novo modelo de atenção se impõe, ditando o ritmo com vistas à atualização nos modos de gestão e em serviços extra-hospitalares. Dentre, destaca-se o Serviço de Residenciais Terapêuticos (SRT) e sua estrutura de atendimento em casas no espaço urbano, constituídas para atender as necessidades dos moradores quanto à moradia, suporte profissional interdisciplinar e inserção social dos internos (Ministério da Saúde, 2004). 
$\mathrm{Na}$ arte de existir nos contextos urbanos das cidades, Lefebvre (2001, p. 22) destaca os "confrontos das diferenças, conhecimentos e reconhecimentos recíprocos dos modos de viver, dos 'padrões' que coexistem". Assim, a reintegração da "loucura" aos espaços urbanos, refletida nos preceitos da Psicologia Ambiental enaltece a visualização do ambiente como elemento componente de subjetividade e que por elas se constitui (Aragonés \& Amérigo, 1998). Nesse viés, esses ambientes pelos quais os "loucos" transitam se tornam palco de desenvolvimento humano (Lima \& Yasui, 2014), o que reforça a importância de se pensar sobre os fatores que influenciam as sensações de prazer e ou desprazer experimentadas em determinados ambientes (Gressler, 2014).

No intuito de explorar as potencialidades dos lugares e averiguar como ocorrem as interações pessoas-ambientes, almeja-se explorar estudos que identifiquem não apenas o espaço por si só, mas que contemplam a qualificação desse, que reflitam sobre os atributos naturais e construídos e os estímulos sensoriais e perceptivos próprios dessas relações. Ao tentar analisar os aspectos físicos e os significados oriundos dos enlaces que as pessoas estabelecem com os espaços, estima-se que se o ambiente emana componentes estressores, tendo em vista as mensagens que comunica, pode-se presumir, positivamente, que igualmente seja capaz de consentir e promover a recuperação do estresse (Felippe et al., 2017). No caso de constituição de cognições de valência positiva, Ulrich et al. (1991) destacam a importância de verificar se tais ambientes estão despertando interesse, aprazibilidade e calma. Ao imergir no contexto urbano, além de considerar as variáveis imersas nos residenciais terapêuticos, trata-se de grande relevância explorar o entorno das casas, a fim de confiar resguardo ao tratamento das pessoas e aos preceitos da Cartilha de Residenciais Terapêuticos (Ministério da Saúde, 2004).

Ao debruçar-se sobre a compreensão do desenvolvimento humano nas cidades, pretende-se contemplar a discussão sobre a saúde mental no enlace com a cultura, com aspectos físicos e simbólicos dos lugares. Nessa vertente, torna-se fundamental ancorar a investigação em parâmetros condizentes às produções científicas já realizadas que enfocam temática similar. Para tanto, amparada nos objeitvos da Cartilha de Serviços de Residenciais Terapêuticos e na lógica de que a interação com os espaços representa um processo ativo de atribuição de sentidos (Ponte, Bomfim, \& Pascual, 2009), algumas inquietações percorrem a mente, carecendo de aprofundações e busca por material científico que dialogue com as mesmas. Desse modo, com a intenção de compreender e delimitar o estado da arte no que tange à tríade desse estudo e alicerçada ao reduto de residenciais terapêuticos, pretende-se revisar sistematicamente produções científicas relacionadas a partir de determinadas orientações metodológicas.

\section{Método}

Caracterizado como uma pesquisa exploratória descritiva, esse estudo almejou identificar características da ocorrência de um fenômeno em um contexto específico de produções científicas. Para tanto, foi realizada uma revisão sistemática em estudos dedicados à investigação da tríade de conceitos aqui propostos e a aplicação dos mesmos em contextos urbanos e de saúde. A natureza dos dados produzidos pela pesquisa foi de abordagem qualitativa, empreendida para o estabelecimento de categorias de análise de modo a atingir o objetivo do estudo.

Desenvolvida de acordo com as diretrizes do Preferred Reporting Itens for Systematic Reviews and Meta-Analyses (Mother, Liberati, Tetzlaff, Altman, The PRISMA Group, 2009; http://www.prismastatement.org/.), essa revisão se baseia na correspondência ao conjunto de 27 itens sobre informações que devem ser claramente descritas no artigo. Para tanto, dois juízes independentes realizaram a identificação e seleção dos artigos em todas as bases de dados de modo simultâneo ao longo do mês de agosto de 2018. Um terceiro avaliador foi ponderado em caso de discordância entre os 
pares para a arbitragem final, o que, por fim, não se mostrou necessário haja vista o consenso entre os dois juízes frente as divergências encontradas.

Foram buscados artigos voltados aos conceitos de "ambiente restaurador", "significado ambiental" e "identidade social urbana", assim como pesquisas que visam descrever suas dimensões, suas especificidades, suas relaçoes e correlações estabelecidas com conceitos e contextos afins. Além disso, considerando o contexto de interesse foi pesquisado, ainda, o termo "serviço de residencial terapêutico". Embora nem todas as palavras-chave utilizadas constem como descritores na seção de Terminologia em Psicologia da Biblioteca Virtual em Saúde, elas foram escolhidas tendo em vista a recorrente frequência com que são utilizadas em artigos nacionais das mesmas temáticas. Além disso, para melhor adequação terminológica na língua inglesa, as mesmas foram pesquisadas em periódicos e artigos específicos da Psicologia Ambiental, base norteadora desse estudo, tais como: Psyecology, Environment \& Behavior e Journal of Environmental Psychology.

Diante da busca e seleção de palavraschave a serem utilizadas, as mesmas foram pesquisadas em trio e, também, divididas em duplas, a fim de facilitar e orientar a busca. Foram escolhidos, além dos termos principais (ambiente restaurador; significado ambiental; identidade social urbana; serviço de residencial terapêutico), palavras adjacentes que são diretamente ligadas às principais no intuito de ampliar o parâmetro de busca e possibilidade de encontrar material afim. Dentre as terminologias agregadas, se tem: "restauração do estresse"; "significado do lugar"; "identidade de lugar"; "casa"; "saúde mental". Para tanto, os operadores lógicos "and" e "or" foram utilizados para combinação dos termos. Os termos escolhidos foram pesquisados em português e na sua respectiva tradução e adequação em inglês.

As buscas contemplaram o levantamento de produções científicas em diferentes bases de dados, de caráter nacional e internacional. As bases nacionais selecionadas foram: Biblioteca Virtual de Psicologia (BVSPSI), The Scientific Electronic Library Online (SciELO), Literatura Latino-americana e do Caribe em Ciências da Saúde (LILACS), e Periódicos Eletrônicos em Psicologia (PePSIC). Quanto às bases de dados internacionais que dão acesso às principais revistas da Psicologia Ambiental, escolheu-se: Science Direct e Sage Journals.

No que tange aos critérios para seleção de produções científicas foram estabelecidos e aplicados os seguintes eixos norteadores: a) texto em inglês, espanhol ou português; b) parâmetro cronológico: 2001 - 2018; c) tratarse de artigo de pesquisa empírica; d) adequação aos objetivos e epistemologia desse estudo; e) possuir texto completo disponível na base acessada. Posterior ao processo de seleção de material, o refinamento do conteúdo obtido seguiu os seguintes passos: 1) organização do material levantado de acordo com os parâmetros e descritores escolhidos e exclusão de duplicatas (439 artigos obtidos); 2) leitura dos resumos e seleção inicial dos artigos relacionando aos objetivos do presente estudo (permaneceram 213 artigos); 3) leitura flutuante dos artigos (obtidos 50 artigos); 4) leitura dos artigos na íntegra para prévia apreciação das categorias de análise (chegou-se ao total de 27 artigos); 5) estruturação das categorias e articulação das produções selecionadas; 6) análise e discussão dos resultados.

Tendo em vista as produções científicas encontradas se optou pela análise por meio de categorias temáticas, definidas após a apreciação do material. Portanto, foram estabelecidas três categorias de análise, alicerçadas aos objetivos desta pesquisa, sendo elas: a) engajamento das pessoas no meio urbano; b) enlaces entre aspectos físicos residenciais com percepções dos usuários; c) associações entre recursos psicofisiológicos e bem-estar.

Importante destacar que a investigação conjunta sobre ambientes restauradores, significado ambiental e identidade social 
urbana aplicada a contexto de saúde mental conserva certo grau de inedistismo. Dos resultados encontrados se observou que nenhum estudo verificou exatamente a tríade de conceitos buscados, principalmente ao considerar o ambiente de interesse deste estudo. Assim, foram incluídos na revisão estudos que correspondessem a um ou mais de um conceito investigado que tenha sido explorado em contexto urbano direcionado a aspectos de habitação, apreciação e inclusão nas cidades. Além desses, contemplou-se pesquisas que focaram em aspectos externos à tríade investigada, mas que foram direcionadas à atenção em saúde mental. Um último adendo aos recortes estratégicos e filtros de seleção diz respeito ao parâmetro temporal resguardado (2001 - 2018), a fim de abarcar o período de promulgação da Lei Antimanicomial (10.216 de 2001) para contemplar estudos que já visualizassem uma nova perspectiva de prática em saúde mental.

\section{Resultados e discussão}

Os resultados encontrados demonstram melhores índices nas publicações dos últimos sete anos, com maior destaque no ano de 2013 $(n=7)$ e lacuna nos anos $2014(n=1)$ e 2015 $(n=1)$. Os vinte e sete artigos selecionados para esta revisão sistemática correspondem à estruturação de três categorias temáticas, as quais conservam especificidades quanto aos objetos e direcionamentos de pesquisa utilizados. Para melhor explicá-las e no intuito de detalhar o material obtido, os resultados serão expressos em subtópicos.

\section{Engajamento das pessoas no meio urbano}

Compõem a primeira categoria os estudos direcionados a compreender, sob distintas perspectivas, a inclusão e o engajamento das pessoas em contextos urbanos. Para tanto, a Tabela 1 visa ilustrar aspectos necessários à identificação do material obtido, assim como facilitar a orientação acerca dos artigos analisados.
Com o propósito de relatar processos de inclusão de moradores de SRT em incursões urbanas, descobertas e novas formas de habitar a cidade, Oliveira (2013) explora a desinstitucionalização na cidade de Viamão/RS. Diante de tal pretensão, o autor imerge nessa transição por meio de acompanhamento terapêutico, destacando que esse percurso contribuiu com achados "espontâneos" oportunizados pelo acaso. Com pretensão similar, Matos e Moreira (2013) buscaram descrever o processo de inclusão social dos moradores de um Residencial no Rio de Janeiro por meio de entrevistas. Para as autoras (2013), o modelo de atenção no Serviço investigado corresponde aos preconizados pela Reforma Psiquiática, além de permitir a aproximação da vivência fora do manicômio e a reconstrução desse sujeito enquanto ser social.

O estudo mais antigo encontrado nessa revisão ocorreu no Reino Unido, quando Fieldhouse (2003) se dedicou a compreender o impacto da interação social de doentes mentais com a produção hortícula e a comunidade do entorno. Por meio de entrevistas e grupo focal, o autor buscou entender como a prática surgiu para que pudesse ser aproveitada de forma mais eficaz por usuários de um serviço de atenção à saúde mental. Com isso, constatou que os participantes descreveram situações restauradoras em relação ao estresse por meio do contato com plantas e com o próprio ambiente verde natural, e que isso, ainda, lhes auxiliou na reconstrução da identidade, da vínculação afetiva pelo apego ao lugar e de uma nova rede social significativa. Nesse sentido, Argiles, Kantorski, Willrich, Antonacci e Coimbra (2013) se direcionaram a conhecer a rede de sociabilidade de usuários do SRT de Alegrete/RS. No entanto, as entrevistas realizadas pelos autores foram aplicadas apenas a profissionais $(\mathrm{n}=6)$ do Serviço que enalteceram potenciais nesse modelo de atenção, tais como: o rompimento da segregação da instituição psiquiátrica e o potencial na reinserção à vida em sociedade. 
Tabela 1

Engajamento das pessoas no meio urbano

\begin{tabular}{|c|c|c|}
\hline Autores (ano) & Título do artigo & Origem \\
\hline $\begin{array}{l}\text { Cabassa, Parcesepe, Nicasio, Baxter, } \\
\text { Tsemberis, \& Lewis-Fernández } \\
(2013)\end{array}$ & $\begin{array}{l}\text { Health and Wellness Photovoice } \\
\text { Project: Engaging Consumers With } \\
\text { Serious Mental Illness in Health Care } \\
\text { Interventions. }\end{array}$ & EUA \\
\hline Fieldhouse (2003) & $\begin{array}{l}\text { The impact of an allotment group on } \\
\text { mental health clients' health, } \\
\text { wellbeing and social networking. }\end{array}$ & Reino Unido \\
\hline $\begin{array}{l}\text { Tabrizian, Baran, } \\
\text { Meentemeyer }(2018)\end{array} \quad$ Smith $\quad \&$ & $\begin{array}{lrr}\text { Exploring perceived } & \text { restoration } \\
\text { potential of } & \text { urban green } & \text { enclosure } \\
\text { through } & \text { immersive } & \text { virtual } \\
\text { environments. }\end{array}$ & EUA \\
\hline Biedenweg, Scott \& Scott (2017) & $\begin{array}{l}\text { How does engaging with nature relate } \\
\text { to life satisfaction? Demonstrating the } \\
\text { link between environment-specific } \\
\text { social experiences and life } \\
\text { satisfaction. }\end{array}$ & EUA \\
\hline $\begin{array}{l}\text { Honold, Beyer, Lakes, \& van der } \\
\text { Meer (2012) }\end{array}$ & $\begin{array}{l}\text { Multiple environmental burdens and } \\
\text { neighborhood-related health of city } \\
\text { residents. }\end{array}$ & Alemanha \\
\hline $\begin{array}{l}\text { Francis, Giles-Corti, Wood \& } \\
\text { Knuiman (2012) }\end{array}$ & $\begin{array}{l}\text { Creating sense of community: The } \\
\text { role of public space. }\end{array}$ & Austrália \\
\hline Manzo (2003) & $\begin{array}{l}\text { For better or worse: Exploring } \\
\text { multiple dimensions of place } \\
\text { meaning. }\end{array}$ & EUA \\
\hline Oliveira (2013) & $\begin{array}{l}\text { Os caminhos da reforma psiquiátrica: } \\
\text { acompanhamento terapêutico, } \\
\text { propagação e contágio na metrópole. }\end{array}$ & Brasil \\
\hline $\begin{array}{l}\text { Argiles, Kantorski, Willrich, } \\
\text { Antonacci \& Coimbra (2013) }\end{array}$ & $\begin{array}{l}\text { Redes de sociabilidade: construções a } \\
\text { partir do serviço residencial } \\
\text { terapêutico. }\end{array}$ & Brasil \\
\hline Matos \& Moreira (2013) & $\begin{array}{l}\text { Serviço residencial terapêutico: o } \\
\text { olhar do usuário. }\end{array}$ & Brasil \\
\hline
\end{tabular}

A fim de explorar as dimensões das relações emocionais que as pessoas estabelecem com os lugares, conhecendo-os e examinando o leque de experiências que criam significado, Manzo (2003) desenhou seu estudo. Nele, a autora sugere a necessidade de incorporar ainda mais a magnitude da experiência humana ao discurso atual sobre as relações entre pessoas e lugares. Os resultados obtidos pela autora indicaram, principalmente, que o significado do lugar se desenvolve a partir de um conjunto de emoções $\mathrm{e}$ experiências, tanto positivas quanto negativas. Mais de dez anos depois, Biedenweg, Scott e Scott (2017) realizaram uma investigação com interesses afins, pois buscaram compreender a importância da diversidade de experiências interacionais das pessoas com diferentes ambientes naturais. Para tanto, os autores aplicaram um questionário online em que os resultados enalteceram a importância de tais ambientes para o bem-estar humano, conectando-o à percepção de satisfação com a vida. Os benefícios da interação humana com espaços públicos também foram averiguados em um estudo quantitativo australiano, no qual Francis, Giles-Corti, Wood e Knuiman (2012) examinaram a relação entre quatro espaços públicos e o senso de comunidade em residentes de novos conjuntos residenciais. Os autores concluíram que não se trata da frequência com que as pessoas acessam, mas sim a qualidade na elaboração de espaços públicos que pode, principalmente, auxiliar e melhorar o senso de comunidade a ser desenvolvido quando há uma transição de habitação. 
No intuito de verificar o discurso de residentes de agências de apoio habitacional nos Estados Unidos, Cabassa et al. (2013) abriram diálogo sobre a saúde física dos moradores a fim de gerar conhecimento e estratégias baseadas na própria comunidade. Por meio de imagens e narrativas se obteve junto aos moradores verbalizações sobre suas visões e preferências quanto à caracterização de intervenções de saúde que poderiam, então, ser efetivamente introduzidas nessas agências com o propósito de reduzir as vulnerabilidades que os caracterizavam. Também dedicados a entender os "convites ambientais" para que as pessoas habitem um espaço, Tabrizian, Baran, Smith e Meentemeyer (2018) procuraram explorar o impacto da percepção de segurança na recuperação do estresse. Para isso, exploraram os efeitos do cercamento do espaço verde sobre a recuperação e a segurança percebida com caminhada em dois parques urbanos. Os autores destacaram a importância de se bem planejar espaço e a configuração dos lugares enquanto meios potencialmente benéficos à saúde, uma vez que o arranjo espacial foi $o$ principal modelador da percepeção de segurança e de restauro psicológico.

Na pesquisa de Honold, Beyer, Lakes, e van der Meer (2012) se destacou a inclusão das variáveis "ruído de tráfego, poluição do ar e falta de espaços verdes públicos" a fim de examinar as relações entre múltiplos ônus e autoavaliação da saúde de residentes da cidade em Berlim. Para isso, analisaram relatos de residentes sobre a satisfação com o bairro e com a vida, o comportamento de saúde e os sintomas psicológicos e de saúde física. Dos reultados obtidos, os pesquisadores salientaram que a satisfação com a vizinhança é o principal fator a ser beneficiado com a ressignificação das variáveis citadas e que os achados desse estudo têm implicações tanto para o planejamento urbano quanto para a saúde pública. Por fim, de modo geral, percebeu-se que o caráter dedicado não apenas à compreensão, mas também à modificação dos espaços para melhoria da saúde humana dita o ritmo dessa categoria e das tendências científicas nos Estudos PessoaAmbiente (EPA).

\section{Enlaces entre aspectos físicos residenciais com percepções dos usuários}

A segunda categoria é composta de estudos interessados em verificar ligações acerca de características físicas de residências alicerçadas por percepções de moradores sobre a relação pessoa-ambiente. Desse modo, a Tabela 2 expõe as pesquisas selecionadas.

Ao buscar examinar os efeitos do ambiente de habitação percebida com o bemestar em pessoas com graves doenças mentais de um programa de suporte habitacional, Wright e Kloos (2007) exploram variáveis ligadas à configuração dos apartamentos, ao bairro e as características sociodemográficas dos moradores. Por meio de entrevistas, observação, levantamento de perfil censitário da comunidade e avaliação de gestão dos ambientes habitacionais os autores constataram que as variáveis do nível de vizinhança são os preditores mais influentes para entender a variação no bem-estar, além das relacionadas à configuração das moradias. Enlaces como esses também foram realizados por Kylén, Schmidt, Iwarsson, Haak e Ekström (2017) ao investigar a associação entre aspectos percebidos do lar com o bem-estar psicológico em pessoas vivendo em comunidade com idade entre 67 e 70 anos. Os autores (2017) entrevistaram 371 residentes e constataram redução de índices depressivos entre os participantes que relataram maior vínculo cognitivo-emocional e social com o lar, e entre aqueles que sentiram que tinham controle sobre sua situação de moradia.

A fim de compreender o papel e significado do lar para cuidadores familiares co-residentes de pessoas com demência, Soilemezi, Drahota, Crossland, Stores e Costall (2017) entrevistaram treze cuidadores. Dentre os resultados obtidos se destacaram os diferentes significados da casa, que vão desde a percepção da mesma como um refúgio seguro até a noção de que ela remete à prisão. Com similar intenção científica, o estudo de Muenchberger, Ehrlich, Kendall e Vit (2012) 
averiguou a relevância de se redefinir o ambiente doméstico conforme as necessidades de seus habitantes. Entrevistas com moradores, familiares e funcionários que trabalham no local foram realizadas para conhecer a importância do lugar para pessoas com deficiências residentes em casas terapêuticas. Sobretudo, a pesquisa enalteceu a importância de espaços para se expressar livremente bem como a projeção dos mesmos para atender às necessidades de locomoção e de cuidado. Ademais, os autores (2012) ressaltaram a estrutura da paisagem terapêutica aplicada nos ambientes, apoiando-se nos benefícios possibilitados, conceito entendido por eles como lugares de tratamento que são o resultado combinado de uma interação entre ambientes construídos, naturais, simbólicos e sociais.

\section{Tabela 2}

Enlaces entre aspectos físicos residenciais com percepções dos usuários

\begin{tabular}{|c|c|c|}
\hline Autores (ano) & $\begin{array}{c}\text { Título do artigo } \\
\end{array}$ & Origem \\
\hline Santos Junior \& Silveira (2009). & $\begin{array}{l}\text { Práticas de cuidados produzidas no } \\
\text { serviço de residências terapêuticas: } \\
\text { percorrendo os trilhos de retorno à } \\
\text { sociedade. }\end{array}$ & Brasil \\
\hline Silveira \& Santos Junior (2011). & $\begin{array}{l}\text { Que eles falem por si: relatos dos } \\
\text { profissionais sobre a experiência nas } \\
\text { residências terapêuticas. }\end{array}$ & Brasil \\
\hline Silva \& Azevedo (2011) & $\begin{array}{l}\text { As novas práticas em saúde mental e } \\
\text { o trabalho no serviço residencial } \\
\text { terapêutico. }\end{array}$ & Brasil \\
\hline Franco \& van Stralen (2015) & $\begin{array}{l}\text { Desinstitucionalização psiquiátrica: } \\
\text { do confinamento ao habitar na cidade } \\
\text { de Belo Horizonte. }\end{array}$ & Brasil \\
\hline Wright \& Kloos (2007) & $\begin{array}{l}\text { Housing environment and mental } \\
\text { health outcomes: A levels of analysis } \\
\text { perspective. }\end{array}$ & EUA \\
\hline $\begin{array}{c}\text { Kylén, Schmidt, Iwarsson, Haak, \& } \\
\text { Ekström (2017) }\end{array}$ & $\begin{array}{l}\text { Perceived home is associated with } \\
\text { psychological well-being in a cohort } \\
\text { aged } 67-70 \text { years. }\end{array}$ & Suécia \\
\hline $\begin{array}{l}\text { Soilemezi, Drahota, Crossland, } \\
\text { Stores \& Costall (2017) }\end{array}$ & $\begin{array}{l}\text { Exploring the meaning of home for } \\
\text { family caregivers of people with } \\
\text { dementia. }\end{array}$ & Reino Unido \\
\hline $\begin{array}{c}\text { Muenchberger, Ehrlich, Kendall \& } \\
\text { Vit (2012) }\end{array}$ & $\begin{array}{l}\text { Experience of place for young adults } \\
\text { under } 65 \text { years with complex } \\
\text { disabilities moving into purpose-built } \\
\text { residential care. }\end{array}$ & Austrália \\
\hline $\begin{array}{c}\text { Shepley, Watson, Pitts, Garrity, } \\
\text { Spelman, Fronsman \& Kelkar (2017) }\end{array}$ & $\begin{array}{l}\text { Mental and behavioral health } \\
\text { settings: Importance \& effectiveness } \\
\text { of environmental qualities \& features } \\
\text { as perceived by staff. }\end{array}$ & EUA \\
\hline Lindal \& Hartig (2013) & $\begin{array}{l}\text { Architectural variation, building } \\
\text { height, and the restorative quality of } \\
\text { urban residential streetscapes. }\end{array}$ & Austrália e Suécia \\
\hline
\end{tabular}

Ainda sobre as carências e cuidados no atendimento em saúde mental, três SRT's foram investigados, um deles por Santos Junior e Silveira (2009) que buscaram compreender a visão de profissionais do Serviço sobre a prática de cuidado produzida. Para tanto, foram realizadas entrevistas, observação sistemática e registro em diário de campo. Os pesquisadores verificaram que o cuidado nesse modelo de atenção é mais humanizado e valoriza a singularidade dos moradores, além de ser um estímulo à prática do autocuidado. Nesse 
sentido, Silva e Azevedo (2011) se dedicaram a investigar a percepção de cuidadores sobre o trabalho desenvolvido em um Residencial de Caicó/RN. Seis profissionais da enfermagem responderam a uma entrevista que resultou na compreensão da morada como ambiente de auxílio à qualidade de vida dos moradores, ainda que também tenham sido denunciadas precariedades no espaço físico, nos recursos materiais e insuficientes profissionais na Residência. Outrossim, Silveira e Santos Junior (2011) ao procurar conhecer as dificuldades enfrentadas pelos profissionais e as perspectivas por eles apontadas para os moradores na prática de cuidado delineada em um SRT, entrevistaram os trabalhadores e observaram que tanto a insuficiência de recursos humanos quanto a falta de capacitação e de apoio social são os principais obstáculos à inserção social dos residentes.

O estudo de Lindal e Hartig (2013) foi desenvolvido a partir de uma avaliação das características físicas específicas de ambientes residenciais urbanos que permitem a restauração psicológica ou se agrupam às preferências ambientais dos moradores. No entanto, foi utilizado sobremaneira o conceito de preferência ambiental enquanto alicerce da restauração do estresse, sob o argumento de que há poucas pesquisas dedicadas ao conceito de ambiente restaurador. Lindal e Hartig (2013) partem da perspectiva de que há fortes associações entre o conceito de preferência ambiental e os aspectos inerentes à experiência restaurativa e, desse modo, adotam a conceituação de Kaplan e Kaplan (1989) sobre preferência ambiental, definindo-a como processo que estimula a exploração e oferece envolvimento imediato com o meio ambiente. Lindal e Hartig (2013) encontraram resultados diversos, mas destacaram congruência a estudos anteriores que demonstram que diferentes ambientes residenciais urbanos oferecem distintas oportunidades de experiência restaurativa.

$\mathrm{Na}$ intenção de explorar o "depois" do mapeamento sobre a importância e a eficácia de qualidades e características ambientais específicas, Shepley et al. (2017) partiram para etapas seguintes, buscando gerar diretrizes que orientem o design e as instalações de espaços de tratamento de saúde mental e comportamental através da criação e testagem de uma ferramenta para avaliar as instalações de saúde mental e comportamental. Em suma, puderam observar que os resultados satisfizeram as pretensões, mas que os estudos posteriores deveriam ampliar escopo para mensurar a importância do apoio da equipe para que o planejamento de espaços promotores do bem-estar considere a importância da estética e ofereça situações cujos efeitos possam ser positivos os usuários do lugar.

\section{Associações entre estresse e bem-estar}

Congregadas na terceira categoria estão as pesquisas dedicadas a discorrer sobre associações entre recursos psicológicos e fisiológicos com o bem-estar humano. Para tanto, a Tabela 3 explana os artigos inerentes a esse enfoque, seguida pela apresentação dos achados.

$\mathrm{Na}$ intenção de comparar efeitos restauradores psicofisiológicos de relaxamento entre ambiente natural e simulado em pessoas com síndrome de Burnout, Kjellgren e Buhrkall (2010) expuseram 18 participantes a 30 minutos de relaxamento em um ambiente natural com uma simulação de interior do mesmo ambiente natural. Os autores constataram que ambos os ambientes facilitaram a redução do estresse, com o ambiente natural trazendo aumento de energia e estados alterados de consciência, possivelmente aumentando e promovendo a restauração. Ainda, em exploração sobre o conceito de ambientes restauradores, Ratcliffe, Gatersleben e Sowden (2013) buscaram compreender em que medida os sons das aves estão relacionados às percepções de restauração da atenção e recuperação em relação ao estresse em ambientes naturais. Para tanto, por meio de entrevistas com residentes em contextos urbanos os autores (2013) se guiaram pela escolha de estímulos naturais auditivos, reconhecendo-os como recursos 
potencialmente benéficos à restauração, sobretudo o som dos pássaros.

Ao pensar no potencial restaurador de ambientes públicos urbanos, dois estudos se prontificaram a analisá-los sob diferentes óticas. Abdulkarim e Nasar (2014) se dedicaram a analisar como fazer praças urbanas mais habitáveis e restauradoras, para tanto utilizaram a Teoria de Recuperação da Atenção de Kaplan e Kaplan (1989) e aplicaram a Escala de Estresse Percebido (PRS 5) para embasar suas descobertas. Os autores constataram que, particularmente, a fascinação e a compatibilidade se relacionaram aos elementos restauradores apontados pelos participantes sobre as praças analisadas. Por outro lado, utilizando a teoria Psicoevolucionista de Ulrich (1984), Gidlow et al. (2016) procuraram verificar respostas psicofisiológicas de pessoas estressadas e não estressadas submetidas a caminhadas em três ambientes distintos. Os participantes foram submetidos a caminhadas de 30 minutos em três ambientes agradáveis: residencial (urbano), natural (verde) e natural com água (azul). Em resumo dos achados científicos, observou-se redução do estresse (mudanças de humor e cortisol) em todos os ambientes, mas com destaque aos ambientes naturais por conferirem benefícios cognitivos adicionais que duraram pelo menos 30 minutos após a saída do ambiente.

Tabela 3

Associações entre recursos psicofisiológicos e bem-estar

\begin{tabular}{|c|c|c|}
\hline Autores (ano) & $\begin{array}{c}\text { Título do artigo } \\
\end{array}$ & Origem \\
\hline Kjellgren \& Buhrkall (2010) & $\begin{array}{l}\text { A comparison of the restorative effect } \\
\text { of a natural environment with that of } \\
\text { a simulated natural environment. }\end{array}$ & Suécia \\
\hline Abdulkarim \& Nasar (2014) & Are livable elements also restorative? & EUA \\
\hline $\begin{array}{l}\text { Ratcliffe, Gatersleben \& Sowden } \\
\text { (2013) }\end{array}$ & $\begin{array}{l}\text { Bird sounds and their contributions to } \\
\text { perceived attention restoration and } \\
\text { stress recovery. }\end{array}$ & Reino Unido \\
\hline Roster, Ferrari \& Jurkat (2016) & $\begin{array}{l}\text { The dark side of home: Assessing } \\
\text { possession 'clutter'on subjective well- } \\
\text { being. }\end{array}$ & EUA \\
\hline $\begin{array}{l}\text { Gidlow, Jones, Hurst, Masterson, } \\
\text { Clark-Carter, Tarvainen, ... \& } \\
\text { Nieuwenhuijsen (2016) }\end{array}$ & $\begin{array}{l}\text { Where to put your best foot forward: } \\
\text { Psycho-physiological responses to } \\
\text { walking in natural and urban } \\
\text { environments. }\end{array}$ & Reino Unido, Finlândia e Espanha. \\
\hline $\begin{array}{l}\text { Francis, Wood, Knuiman \& Giles- } \\
\text { Corti (2012) }\end{array}$ & $\begin{array}{l}\text { Quality or quantity? Exploring the } \\
\text { relationship between Public Open } \\
\text { Space attributes and mental health in } \\
\text { Perth, Western Australia. }\end{array}$ & Austrália \\
\hline Kam \& Siu (2010) & $\begin{array}{l}\text { Evaluation of a horticultural activity } \\
\text { programme for persons with } \\
\text { psychiatric illness. }\end{array}$ & China \\
\hline
\end{tabular}

Com o propósito de verificar a relação entre os atributos de espaços públicos urbanos (ou seja, quantidade e qualidade) e melhor saúde mental (baixo risco de sofrimento psicológico) em residentes de novos conjuntos habitacionais, Francis, Wood, Knuiman e Giles-Corti (2012) fizeram um levantamento com 911 participantes. Os autores constataram que a qualidade dos espaços públicos urbanos dentro de uma vizinhança parece ser mais importante do que a quantidade. Desse modo, se enaltece a perspectiva de que a existência de espaços verdes não necessariamente está alicerçada ao aumento de bem-estar ou qualidade de vida. Nesse viés, Kam e Siu (2010) realizaram um estudo piloto a fim de investigar o efeito da aplicação da atividade hortícola sobre o estresse, desempenho no trabalho e qualidade de vida em pessoas com doença psiquiátrica em uma comunidade chinesa. As constatações 
iniciais do estudo demonstraram que a terapia hortícola se mostrou eficaz na diminuição dos níveis de ansiedade, depressão e estresse entre os participantes.

Por fim, na busca por associações num contexto doméstico, Roster, Ferrari e Jurkat (2016) verificaram o impacto potencialmente prejudicial da desordem tanto no bem-estar psicológico quanto no bem-estar subjetivo. Para isso, entenderam como "desordem" a superabundância de posses que coletivamente criam espaços vivos caóticos e desordenados. Por sua vez, o "lar" foi compreendido como um reflexo da necessidade de se identificar com um ambiente físico. Participantes dos Estados Unidos e Canadá responderam a um questionário online que destacou como pontos positivos o apego ao lugar, ao passo em que a desordem se mostrou negativa à percepção do lar e prejudicial ao bem-estar subjetivo.

\section{Síntese das categorias de análise}

Analisados os materiais obtidos após o último filtro de seleção em que restaram os vinte e sete estudos aqui dispostos, puderam ser apreciadas pesquisas de diferentes localizações geográficas, perspectivas culturais, áreas de estudo e lócus de investigação. Essa heterogeneidade permitiu contemplar e trazer à luz enfoques epistemológicos e metodológicos distintos, porém recheados de descobertas e potenciais recursos a serem replicados.

Os estudos analisados direcionados à tríade conceitual investigada derivaram, predominantemente, dos Estados Unidos $(\mathrm{n}=$ 8), Reino Unido ( $n=4)$ e Austrália $(n=4)$. No entanto, outros grandes centros com pesquisas consolidadas sobre os EPA se fizeram presentes nesse recorte temático, sendo eles: Espanha, Finlândia, Suécia e China. Sobre o cenário brasileiro o que se observou foi maior incidência de artigos oriundos da região sudeste e nordeste, além de dois estudos da região sul. Nota-se, com isso, significativa exploração em lugares cujas regiões possuem maior quantidade e estrutura nos Serviços de Residenciais Terapêuticos. De acordo com o levantamento do Ministério da Saúde sobre os dados acerca da Saúde Mental no país em dezembro de 2014, observa-se que 610 SRT's estavam registrados no momento da coleta. Destes, 122 alocados no nordeste, 20 no centrooeste, 2 no norte, 431 no sudeste e 36 na região sul do Brasil (Ministério da Saúde, 2015). No documento divulgado, o próprio Ministério da Saúde destaca, ainda, o processo evolutivo dos Residenciais e a estatística transitória dos dados, haja vista que atualmente esses dispositivos de atenção estão passando pelo Cadastro Nacional dos Estabelecimentos de Saúde (CNES), o que resulta em dados aproximados e não exatos sobre a realidade.

A abordagem adotada nos estudos verificados se dividiu entre qualitativos e quantitativos. No entanto, quanto às técnicas e instrumentos utilizados se observou predomínio do uso de entrevistas $(n=14)$, sendo que as outras estratégias adotadas percorreram uso de escalas, grupo focal, questionários, etnografia, análise demográfica do ambiente físico, diário de campo e seleção de fotografias. Dos objetivos e perspectivas investigativas em comum, doze delas destacaram a busca de conhecimento das estratégias de melhora da saúde junto da comunidade, assim como a avaliação das características do entorno sociofísicos e das interações comunitárias possíveis nos diferentes espaços. Ainda, se salienta a verificação das características do lar $(n=5)$, comparação de ambientes $(n=3)$, percepções sobre a estrutura de atenção nos serviços de saúde mental $(\mathrm{n}=4)$, assim como a identificação da influência dos elementos naturais na saúde $(\mathrm{n}=3)$. Desse modo, entendeu-se que, embora as categorias tenham seus escopos delimitados por direcionamentos de pesquisa, os resultados se comunicam entre si haja vista a comum perspectiva de investir na relação pessoa-ambiente com base na promoção de saúde.

\section{Considerações finais}

Dentre os Estudos Pessoa-Ambiente (EPA) há poucas pesquisas dedicadas a abordar diretamente as características físicas de 
ambientes urbanos construídos que afetam positivamente a experiência restaurativa (Hidalgo, Berto, Galindo \& Getrevi, 2006). Uma hipótese ponderada por Lindal e Hartig (2013) é a de que esta preferência se associa aos julgamentos de maior probabilidade de restauração em espaços verdes em detrimento dos construídos. Os estudos encontrados nesta revisão corroboram aos achados científicos relacionados à necessidade de se investir na relação pessoa-ambiente, particularmente no bem-estar subjetivo e na qualidade de vida em habitar um espaço. $\mathrm{Na}$ medida em que a experiência vivenciada no universo urbano das cidades causa sobrecarga às pessoas pelo excesso de estímulos aos quais são expostas, o modo de viver das pessoas se adapta constantemente a tais transições tendo em vista as taxas de urbanização crescentes. Em consonância, os estudos do estresse por influência ambiental acompanham os movimentos científicos que reforçam uma maior preocupação em relação às condições de bem-estar e qualidade de vida das pessoas, assim como o modo pelo qual o ambiente pode impactar na saúde humana por meio de alterações dos níveis de estresse (Felippe, 2015; Hartig, 2011).

Ao analisar possibilidades de intervenção em contextos de saúde por meio da aplicação dos conceitos da tríade investigada, traz-se à luz estudos que dão suporte a crescente atenção aos determinantes sociais e ambientais que destacam importância no uso de intervenções multiníveis para melhorar resultados de saúde, particularmente relevante em populações vulneráveis (Braveman, Egerter, \& Williams, 2011; Cabassa et al., 2013). A forte associação entre resultados de saúde e o ambiente físico em que uma pessoa vive ou recebe tratamento vem sendo fonte de inquietação científica há longo tempo (Rollins, 2004; Ulrich et al., 2008; Mourshed \& Zhao, 2012), reforçando, assim, a importância de se averiguar a capacidade ambiental de diferentes lugares na promoção e restauração da saúde e ao estudo do estresse por influência ambiental.

Apesar da exploração do potencial restaurativo de ambientes urbanos ser interesse de pesquisa de alguns dos estudos aqui explorados (Kjellgren \& Buhrkall, 2010; Gidlow et al., 2016; Ratcliffe, Gatersleben \& Sowden, 2013), os EPA ainda não contemplaram, suficientemente, de que modo as características socioambientais específicas de espaços de atenção à saúde podem contribuir para a restauração psicológica do estresse. Nesse sentido, acrescenta-se às lacunas científicas percebidas investigações dedicadas, sobretudo, à verificação de significados ambientais, bem como as interessadas em examinar o conceito de identidade social urbana. Embora os investimentos direcionados aos ambientes restauradores ainda estejam em expansão, os dedicados à apreciação dos conceitos de identidade social urbana e de significado ambiental carecem sobremaneira de atualização científica. Desse modo, sugerem-se aprofundamentos futuros a respeito da tríade conceitual empreendida e seus modelos teóricos em diferentes lócus de atenção à saúde (níveis primários, secundários e terciários). Ao considerar as especificidades de cada ambiente e o cuidado com a saúde humana, torna-se particularmente importante promover embasamento consistente e estimular o fortalecimento das discussões científicas relacionadas à temática.

Entende-se que a presente revisão possibilitou observar, ainda, resguardando as limitações e filtros estabelecidos, que nenhum estudo que investigue ambientes restauradores, significado ambiental e identidade social urbana em contextos de saúde mental foi encontrado. No entanto, os artigos selecionados possuem rico potencial científico e se configuram como relevantes suportes teóricometodológicos para a temática explorada. Estima-se que a investigação de tais conceitos no reduto de atenção à saúde têm significativas chances de se configurar como estudo inédito. Desse modo, pretende-se fomentar base científica dedicada a contribuir com a Psicologia Ambiental e da Saúde, além de apontar indicadores que subsidiem a avaliação e a estruturação de ações tendo em vista as potencialidades do campo de investigação para 
a elaboração de políticas públicas e

planejamentos ambientais.

\section{Referências}

Abdulkarim, D., \& Nasar, J. L. (2014). Are livable elements also restorative?

Journal of Environmental Psychology, 38, 29-38. doi:

10.1016/j.jenvp.2013.12.003

Aragonés, J. I., \& Amérigo, M. (1998).

Psicología Ambiental. Madrid:

Ediciones Pirámide.

Argiles, C. T. L., Kantorski, L. P., Willrich, J. Q., Antonacci, M. H., \& Coimbra, V. C.

C. (2013). Redes de sociabilidade:

construções a partir do serviço residencial terapêutico. Ciência \& Saúde Coletiva, 18(7), 2049-2058. doi: $\underline{\text { 10.1590/S1413-81232013000700020 }}$

Biedenweg, K., Scott, R. P., \& Scott, T. A. (2017). How does engaging with nature relate to life satisfaction? Demonstrating the link between environment-specific social experiences and life satisfaction. Journal of Environmental Psychology, 50, 112-124. doi: 10.1016/j.jenvp.2017.02.002

Braveman, P., Egerter, S., \& Williams, D. R. (2011). The social determinants of health: coming of age. Annual review of public health, 32, 381-398.

Cabassa, L. J., Parcesepe, A., Nicasio, A., Baxter, E., Tsemberis, S., \& LewisFernández, R. (2013). Health and wellness photovoice project: Engaging consumers with serious mental illness in health care interventions. Qualitative Health Research, 23(5), 618-630. doi: $10.1177 / 1049732312470872$

Corraliza, J. A. (1998). Emoción y ambiente. In A. Y. \& A. M. (Eds.), Psicologiía Ambiental (pp. 59-76). Madrid: Pirámide.

Felippe, M. L., Kuhnen, A., Silveira, B. B., \& Lelli, G. (2017). What Is a Restorative Hospital Environment? Environmental Meaning, Affective Stress Restoration and Physical Attributes in Pediatric Inpatient Rooms. Children, Youth and
Environments, 27(1), 17-46. doi: 10.7721/chilyoutenvi.27.1.0017

Felippe, M. L. (2015). Ambiente fisico e linguaggio ambientale nel processo di rigenerazione affettiva dallo stress in camere di degenza pediatrica (Tese de Doutorado). Departamento de Arquitetura e Urbanismo, Università degli Studi di Ferrara, Itália.

Fieldhouse, J. (2003). The impact of an allotment group on mental health clients' health, wellbeing and social networking. British Journal of Occupational Therapy, 66(7), 286-296. doi: 10.1177/030802260306600702

Francis, J., Giles-Corti, B., Wood, L., \& Knuiman, M. (2012). Creating sense of community: The role of public space. Journal of Environmental Psychology, 32(4), 401-409. doi: 10.1016/j.jenvp.2012.07.002

Francis, J., Wood, L. J., Knuiman, M., \& Giles-Corti, B. (2012). Quality or quantity? Exploring the relationship between Public Open Space attributes and mental health in Perth, Western Australia. Social Science and Medicine, 74(10), 1570-1577. doi: 10.1016/j.socscimed.2012.01.032

Gidlow, C. J., Jones, M. V., Hurst, G., Masterson, D., Clark-Carter, D., Tarvainen, M. P., ... Nieuwenhuijsen, M. (2016). Where to put your best foot forward: Psycho-physiological responses to walking in natural and urban environments. Journal of Environmental Psychology, 45, 22-29. doi: 10.1016/j.jenvp.2015.11.003

Gressler, S. C. (2014). O descanso e a teoria dos ambientes restauradores. Recuperado de http://repositorio.unb.br/handle/10482/1 $\underline{5845}$

Hartig, T. (2011). Issues in restorative environment research: matters of 
measurement. In: B. Fernández-

Ramírez, C. H., Villodres, C. M. S., Ferrer, M. J. M., Méndez (Orgs.), Psicología Ambiental 2011: entre los estudios urbanos y el análisis de la sostenibilidad (pp. 41-66). Universidad de Almería: Associación de Psicologia Ambiental (PSICAMB).

Hartig, T., \& Staats, H. (2005). Linking preference for environments with their restorative quality. In From landscape research to landscape planning: Aspects of integration, education and application. (pp. 279-292).

Honold, J., Beyer, R., Lakes, T., \& van der Meer, E. (2012). Multiple environmental burdens and neighborhood-related health of city residents. Journal of

Environmental Psychology, 32(4), 305317. doi: 10.1016/i.jenvp.2012.05.002

Kam, M. C. Y., \& Siu, A. M. H. (2010). Evaluation of a horticultural activity programme for persons with psychiatric illness. Hong Kong Journal of Occupational Therapy, 20(2), 80-86. doi: 10.1016/S1569-1861(11)70007-9

Kaplan, R., \& Kaplan, S. (1989). The experience of nature: A psychological perspective. (C. U. Press, Ed.).

Kjellgren, A., \& Buhrkall, H. (2010). A comparison of the restorative effect of a natural environment with that of a simulated natural environment. Journal of Environmental Psychology2, 30, 464472.

Kylén, M., Schmidt, S. M., Iwarsson, S., Haak, M., \& Ekström, H. (2017). Perceived home is associated with psychological well-being in a cohort aged 67-70 years. Journal of

Environmental Psychology, 51, 239247. doi: $10.1016 /$ j.jenvp.2017.04.006

Lefebvre, H. (2001). O direito à cidade $\left(5^{\mathrm{a}}\right)$. São Paulo: Centauro.

Lima, E. M. F. A., \& Yasui, S. (2014). Territórios e sentidos: espaço, cultura, subjetividade e cuidado na atenção psicossocial. Saúde Em Debate, 38(102), 593-606. doi: $10.5935 / 0103-$ $\underline{1104.20140055}$
Lindal, P. J., \& Hartig, T. (2013).

Architectural variation, building height, and the restorative quality of urban residential streetscapes. Journal of Environmental Psychology, 33, 26-36. doi: 10.1016/j.jenvp.2012.09.003

Manzo, L. (2003). Beyond house and haven: toward a revisioning of emotional relationship with places. Journal of Environmental Psychology, 23, 47-61.

Matos, B. G. de, \& Moreira, L. H. de O. (2013). Serviço residencial terapêutico: o olhar do usuário. Escola Anna Nery Revista de Enfermagem, 17(4), 668-676. doi: $\underline{10.5935 / 1414-8145.20130010}$

Ministério da Saúde. Secretaria de Atenção à Saúde. Departamento de Ações Programáticas e Estratégicas. (2004). Residências terapêuticas: o que são, para que servem? Brasília, DF.

Ministério da Saúde. SAS/DAPES. Coordenação Geral de Saúde Mental, Álcool e Outras Drogas. Saúde Mental em Dados - 12, Ano 10, $\mathrm{n}^{\circ}$ 12, outubro de 2015. Brasília, DF.

Mourão, A. R. T., \& Cavalcante, S. (2006). O processo de construção do lugar e da identidade dos moradores de uma cidade reinventada. Estudos de Psicologia, 11(2), 143-151.

Mourshed, M., \& Zhao, Y. (2012). Healthcare providers' perception of design factors related to physical environments in hospitals. Journal of Environmental Psychology, 32(4), 362-370.

Muenchberger, H., Ehrlich, C., Kendall, E., \& Vit, M. (2012). Experience of place for young adults under 65 years with complex disabilities moving into purpose-built residential care. Social Science and Medicine, 75(12), 21512159. doi: 10.1016/j.socscimed.2012.08.002

Oliveira, R. W. (2013). The psychiatric reform ways: Therapeutic assistance and propagation in metropolis . Psicologiae Sociedade, 25(SPECIALISSUE 2), 90 94. Retrieved from http://www.openpsychodynamic.com/?p age $\mathrm{id}=32$ 
Pol, E. (1996). La apropiación del espacio. Cognición, representación y apropiación del espacio, 45-62.

Ponte, A. Q., Bomfim, Z. Á. C., \& Pascual, J. G. (2009). Considerações Teóricas Sobre Identidade De. Psicologia Argumento, 27(59), 345-354.

Rapoport, A. (1990). Levels of meaning and types of environments. In Y. Yoshitake et al,. (Eds.), Current Issues in Environmental-Behavior Research, Proceedings of the Third Japan-US Seminar, University of Tokyo, Kyoto, Tokyo (pp. 135-147).

Ratcliffe, E., Gatersleben, B., \& Sowden, P. T. (2013). Bird sounds and their contributions to perceived attention restoration and stress recovery. Journal of Environmental Psychology, 36, 221228. doi: 10.1016/i.jenvp.2013.08.004

Rollins, J. A. (2004). Evidence-based hospital design improves health care outcomes for patients, families, and staff. Pediatric Nursing, 30, 338-339.

Roster, C. A., Ferrari, J. R., \& Jurkat, M. (2016). The dark side of home: Assessing possession "clutter" on subjective well-being. Journal of Environmental Psychology, 46, 32-41. doi: 10.1016/j.jenvp.2016.03.003

Santos Junior, H. P. de O. S., \& Silveira, M. de F. de A. (2009). Práticas de cuidados produzidas no serviço de residências terapêuticas : percorrendo os trilhos de retorno à sociedade. Revista Escola de Enfermagem USP, 43(4), 788-795.

Shepley, M. M. C., Watson, A., Pitts, F., Garrity, A., Spelman, E., Fronsman, A., \& Kelkar, J. (2017). Mental and behavioral health settings: Importance \& effectiveness of environmental qualities $\&$ features as perceived by staff. Journal of Environmental Psychology, 50, 3750. doi: $\underline{10.1016 / \text { j.jenvp.2017.01.005 }}$

Silva, D. S., \& Azevedo, D. M. (2011). As novas práticas em saúde mental e o trabalho no serviço residencial terapêutico. Escola Anna Nery, 15(3), 603-609. doi: 10.1590/S1414$\underline{81452011000300023}$
Silveira, M. de F. de A., \& Santos Junior, H. P. de O. (2011). Que eles falem por si: relatos dos profissionais sobre a experiência nas residências terapêuticas. Ciência \& Saúde Coletiva, 16(4), 20892098. doi: $\underline{10.1590 / S 1413-}$ 81232011000400008

Soilemezi, D., Drahota, A., Crossland, J., Stores, R., \& Costall, A. (2017). Exploring the meaning of home for family caregivers of people with dementia. Journal of Environmental Psychology, 51, 70-81. doi: 10.1016/j.jenvp.2017.03.007

Tabrizian, P., Baran, P. K., Smith, W. R., \& Meentemeyer, R. K. (2018). Exploring perceived restoration potential of urban green enclosure through immersive virtual environments. Journal of Environmental Psychology, 55, 99-109. doi: $10.1016 /$ j.jenvp.2018.01.001

Ulrich, R. S. (1984). View through a window may influence recovery from surgery. Science, 224(4647), 420-421. doi: 10.1126/science.6143402

Ulrich, R. S., Zimring, C., Zhu, X., DuBose, J., Seo, H., Choi, Y., et al. (2008). A review of the research literature on evidence-based healthcare design (part I). Health Environments Research and Design, 1, 61-125.

Ulrich, R. S., Simons, R. F., Losito, B. D., Fioritom, E., Miles, M. A., \& Zelson, M. (1991). Stress recovery during exposure to natural and urban environments. Journal of Environmental Psychology, 11(3), 201-230. doi: 10.1016/S02724944(05)80184-7

Valera, S., \& Pol, E. (1994). El concepto de identidad social urbana: una aproximación entre la psicología social y la psicología ambiental. Anuario de psicología/The UB Journal of psychology, (62), 5-24.

Wright, P. A., \& Kloos, B. (2007). Housing environment and mental health outcomes: A levels of analysis perspective. Journal of Environmental Psychology, 27(1), 79-89. doi: 10.1016/j.jenvp.2006.12.001 
Yasui, S. (2010). Rupturas e encontros:

desafios da reforma psiquiátrica brasileira.

SciELO-Editora FIOCRUZ

\section{Dados sobre os autores:}

- Bettieli Barboza da Silveira: Psicóloga, mestre em Psicologia e atualmente cursa doutorado em Psicologia na Universidade Federal de Santa Catarina (UFSC). Membro do Laboratório de Psicologia Ambiental da UFSC. Atualmente, desenvolve sua tese de doutorado no âmbito da psicologia ambiental e da saúde mental, investigando o Serviço Residencial Terapêutico em Santa Catarina.

- Ariane Kuhnen: Psicóloga, Mestre em Sociologia Política e Doutora em Ciências Humanas. Professora titular do Departamento de Universidade Federal de Santa Catarina (UFSC). Coordena o Laboratório de Psicologia Ambiental da UFSC, onde orienta pesquisas sobre os Estudos Pessoa-Ambiente. 